Books, videos, CD-ROMs, DVDs and any

other relavent items submitted for a review

in the $B D J$ should be addressed to: Kate

Maynard, Assistant Editor, British Dental

Journal, NPG, 4-6 Crinan Street, London, N1 9XW
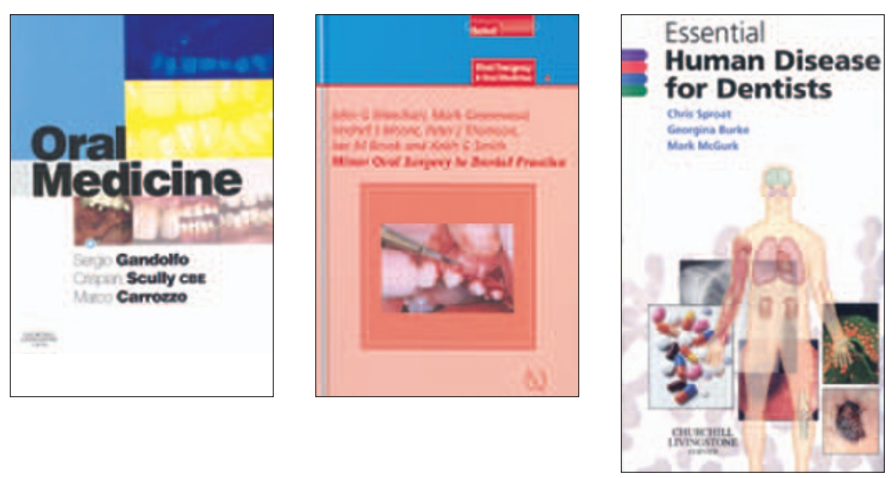

as oral cavity cancers may not have

Unfortunately the authors of the present work seem to have been let down by their publisher in this regard.

The teaching of oral medicine has long been complicated by the absence of good textbooks at undergraduate and specialist level. Most existing texts are too synoptic, or contain material that is controversial or the personal opinions of the authors, or have clinical illustrations that are not of adequate quality. I had hoped that this might be the text that would fill the gap in the market. Unfortunately it is not. I would be reluctant to recommend this text to undergraduates and would only recommend it to trainees as a supplementary text.

B. McCartan been better. For example, under 'other gingival hyperplasia' the reader is referred to Crohn's disease. However, the entry for the latter has no information on the gingival changes that might be expected. Similarly, the captions to some figures are inconsistent with the text. It is also rather surprising in an oral medicine text to find no entries for trigeminal neuralgia or orofacial granulomatosis. A colourcoding scheme differentiates conditions according to the level of expertise required for management. This is a clever and useful scheme. There are a number of good algorithms on the management of oral conditions. That for recurrent aphthous stomatitis seems to suggest that if the condition is recurrent (which is self-evident) then treatments such as thalidomide should be considered. This is sloppy editing and potentially dangerous in a text aimed at, amongst others, family doctors. I would be concerned that the section on drugs for the treatment of mucosal disease presents a number of agents in various groups without any strong sense of whether or not they would be appropriate first line treatments.

As much of the diagnostic process for diseases of the oral mucous membranes depends on a close evaluation of colour changes in the tissues, accurate colour rendition is essential in the illustrations; a good example is the excellent atlas of oral diseases that Professor Scully co-authors.

\section{Minor oral surgery in dental practice}

J. G. Meechan, M. Greenwood, U. J. Moore, P. J. Thomson, I. M. Brook, K. G. Smith

UK: Quintessence

price $€ 28.00$, pp 124

ISBN 1850970823

This is a generally well-written and illustrated small hard backed book, which gives a broad overview of minor oral surgery (MOS). It is divided into 14 chapters, covered over 168 pages. In general the book is easy to read, with aims and outcomes clearly stated at the beginning of each chapter, to ensure that the reader has understood the contents. Introductory chapters on patient assessment, the general principles of MOS and pharmacology give a broad overview of diagnosis and treatment planning. All of the chapters are readily readable, with the one on surgical endodontics being especially enjoyable.

One of the major criticisms is that the book does not make clear exactly what procedures can be appropriately carried out in a general dental practice setting. For example, in the chapter on biopsies it was stated that only 'lesions that appear malignant should not be biopsied in the general practice setting'. This is worrying typical presentations. Therefore we would strongly caution the dental practitioner in following the advice given in this chapter. Another example was how to manage a fractured tuberosity - no guidelines were given about when the patient should be referred or when the tuberosity should be removed.

The specific pre-operative assessment of surgical procedures that could safely be performed in a dental practice setting was not directly addressed. For example, in the chapter on impacted teeth, although the NICE guidelines were documented for the removal of third molars, there was no method given to enable a reader to adequately assess the degree of difficulty that might be encountered with this procedure. Without this knowledge, a practitioner might get into considerable difficulty in attempting a procedure that is then found to be beyond their level of expertise. Diagrams showing the features likely to increase the difficulty of surgery and/or a table of possible complex cases would have been beneficial.

In general, this book should be of interest to undergraduate dental students, and postgraduate practitioners who wish to update their knowledge or gain some insight into the range of MOS procedures. However, it is should be made clear early in the book that many of the procedures described would not usually be performed in a general dental practice setting. As long as this is borne in mind, this book can be recommended to the dental practitioner.

P. A. Brennan

Essential human disease for dentists

\author{
C. Sproat, G. Burke, M. McGurk \\ UK: Elsevier \\ price £24.99, pp 311 \\ ISBN 0443100985
}

It is not uncommon to provide dental treatment for patients who have been diagnosed with a significant medical 
condition. In many circumstances this can usually be provided safely in a general dental practice setting. However it is imperative that a dentist should have a sound basis of common medical conditions and their relevance in dentistry. The aims of this book include acting as a revision aid for undergraduates, a chairside reference and to describe commonly occurring medical conditions which are encountered in general dental practice.

This textbook is divided into 23 chapters. The authors and the contributors have a range of experiences involving both medical and surgical specialities. A reassuring factor is the presence of dual-qualified authors (medical and dental qualifications). This is apparent when reading the textbook and it is clear that the authors have taken measures to ensure that each chapter is dentally relevant. The majority of the chapters describe a particular medical system ie cardiovascular. This is then further subdivided into individual conditions affecting that system ie hypertension. Each condition is then described systematically in a set clear format which describes the epidemiology, aetiology, pathogenesis, clinical features, diagnosis and treatment of that condition. The dental relevance of that condition is then described in a highlighted coloured box at the end of each section which is easy to locate quickly and understand. Additional chapters include assessment and risk management, elderly patients, disabled patients, pregnancy, hospital patients, health promotion and medical emergencies in dental practice.

This textbook is compact in size and can be easily stored in an individual dental surgery or carried on a daily basis. The overall presentation has a modern approach which enhances its readability. The text is concise and has been written with clarity, both of which help to provide the essential points in a fashion which is easy to memorise. This book has excellent clinical photographs and line diagrams which support the general text.

In summary this is an excellent book which is highly recommended to general dental practitioners, undergraduates, DCP and postgraduate students. This book easily satisfies its aims and objectives. The authors have managed to produce a textbook which is written primarily for dentists thus appreciating the possible implications of medical conditions when providing dental treatment. It provides information on complex medical conditions in an easy to understand fashion which can be rapidly accessed.

J. Seehra

\section{Flexible working and training for doctors and dentists}

\section{A. Hastie (Ed) \\ UK: Radcliffe \\ price $€ 24.95$, pp 224 \\ ISBN 1846190258}

The aim of this book is to explain the regulations relating to part-time and

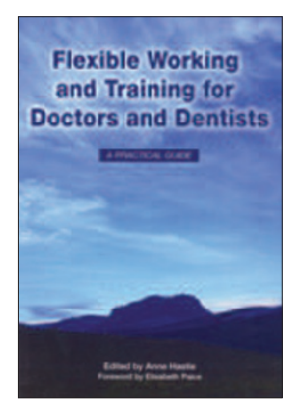

flexible training for doctors and dentists in the UK. Several authors have contributed to the text, and the result is a comprehensive guide to current practice. The information given is up to date and well researched within its context, but the book relates mainly to the training requirements for medical doctors, and females at that. Many references are made to the shortcomings of the current system of training as it relates to women wishing to take time out for family reasons, but the authors do not give a similar emphasis to problems of training for male students.

For the dentist, there is one main chapter pertaining to the regulations for vocational training, and brief references to dentistry in others, however the bulk of the book describes in detail the rules for flexible medical training, either in primary, secondary or academic disciplines. The authors of chapters which give information aimed at both doctors and dentists show limited knowledge of regulations for general dental practice, and those describing academic and hospital based careers tend to refer to dental training in broad terms only.

The later chapters do provide information on pensions, maternity leave and work overseas and there is helpful information on disability, but the dentist wishing to use the book to research a training pathway would need to read through the majority of the text before finding relevant details, and may be left with many unanswered questions.

I. M. Madden 